\title{
Spectroscopy of Neutron-Deficient Nuclei Near the Z=82 Closed Shell via Symmetric Fusion Reactions
}

\author{
F.G. Kondev ${ }^{1, a}$, M.P. Carpenter ${ }^{1}$, S. Zhu' ${ }^{1}$, R.V.F. Janssens ${ }^{1}$, I. Ahmad ${ }^{1}$, B.B. Back ${ }^{1}$, P.F. Bertone ${ }^{1}$, J. Chen ${ }^{1}$, C.J. \\ Chiara $^{1,2}$, C.A. Copos $^{1}$, J.P. Greene ${ }^{1}$, C.R. Hoffman ${ }^{1}$, B.P. Kay ${ }^{1}$, T.L. Khoo ${ }^{1}$, T. Lauritsen ${ }^{1}$, E.A. McCutchan ${ }^{1, b}$, C. \\ Nair $^{1}$, A.M. Rogers ${ }^{1, c}$, D. Seweryniak ${ }^{1}$, and D. J. Hartley ${ }^{3}$ \\ ${ }^{1}$ Argonne National Laboratory, Argonne, Illinois 60439, USA \\ ${ }^{2}$ Department of Chemistry and Biochemistry, University of Maryland, College Park, Maryland 20742, USA \\ ${ }^{3}$ Department of Physics, U.S. Naval Academy, Annapolis, Maryland 21402, USA
}

\begin{abstract}
In-beam and decay-spectroscopy studies of neutron-deficient nuclei near the $\mathrm{Z}=82$ shell closure were carried out using the Fragment Mass Analyzer (FMA) and the Gammasphere array, in conjunction with symmetric fusion reactions and the Recoil Decay Tagging (RDT) technique. The primary motivation was to study properties of ${ }^{179} \mathrm{Tl}$ and ${ }^{180} \mathrm{Tl}$, and their daughter, and grand-daughter isotopes. For the first time, in-beam structures associated with ${ }^{179} \mathrm{Tl}$ and ${ }^{180} \mathrm{Tl}$ were observed, as well as $\gamma$ rays associated with the ${ }^{180} \mathrm{Tl} \alpha$ decay. No long-lived isomer was identified in ${ }^{180} \mathrm{Tl}$, in contrast with the known systematics for the heavier odd-odd $\mathrm{Tl}$ isotopes.
\end{abstract}

\section{Introduction}

Systematic nuclear structure studies of proton-rich $\mathrm{Au}$, $\mathrm{Hg}, \mathrm{Tl}$, and $\mathrm{Pb}$ nuclei are important in order to elucidate their shape evolution with neutron number from wellstudied deformed minima at mid-shell to the near spherical ground states observed for nuclei near the proton drip line [1]. In addition, detailed knowledge of level and decay properties of those nuclei is also relevant for a better understanding of rare decay modes in this region, such as electron-capture delayed fission [2, 3].

Over the last several years, we have performed a number of experiments using the Gammasphere spectrometer and the Fragment Mass Analyzer (FMA) at ATLAS aimed at measuring properties of proton-rich nuclei in this region (see, for example, Refs. [4, 5] and references therein). In those studies, the use of the FMA was essential in order to differentiate evaporation residues associated with the nucleus of interest from both the large fission background, dominating the reaction cross section, and evaporation residues produced in other reaction channels. The use of symmetric fusion reactions at bombarding energies near the Coulomb barrier proved beneficial in performing these studies. On the one hand, the large, negative $\mathrm{Q}$ values for such reactions [6] result in a relatively low excitation energy of the compound system. As a consequence, the fission competition is reduced and the large fragmentation of the evaporation-residue cross sections is

\footnotetext{
a e-mail: kondev@anl.gov

b present address: NNDC, Brookhaven National Laboratory

${ }^{c}$ present address: Nuclear Science Division, Lawrence Berkeley National Laboratory
}

significantly suppressed, since only a few reaction channels are energetically possible. On the other hand, the enhancement of the fusion cross sections in the sub-barrier region [7] results in relatively large cross sections (up to tens of millibarns) for one- and two-particle evaporation channels, hereby enabling detailed spectroscopy studies to be carried out.

Here, we present new results from spectroscopy studies of the odd-odd ${ }^{180} \mathrm{Tl}$ nuclide. The results on ${ }^{179} \mathrm{Tl}$ are presented elsewhere [8]. The ${ }^{180} \mathrm{Tl}$ nuclide was discovered by Lazarev et al. [2] via the observation of $\mathrm{EC} / \beta^{+}$-delayed fission decays and new results on this decay mode were recently reported by Andreyev et al. [3]. Its main decay mode (94(4) \%) is EC/ $\beta^{+}$decay, which was recently studied by Elseviers et al. [9]. Results from $\alpha$-decay spectroscopy of ${ }^{180} \mathrm{Tl}$ were reported by Toth et al. [10], but the decay scheme remains incomplete, partially owing to the small $\alpha$-decay branch $\left(b_{\alpha}=6(4) \%\right)$. Information on excited structures in ${ }^{180} \mathrm{Tl}$ is also scarce and only three $\gamma$ rays with energies of 89 (1) $\mathrm{keV}, 124(1) \mathrm{keV}$ and 449(1) $\mathrm{keV}$ were assigned to this nuclide in $\alpha$-decay studies of ${ }^{184,184 m} \mathrm{Bi}[11]$.

\section{Experimental Details}

The ${ }^{180} \mathrm{Tl}$ isotope was produced in the $1 \mathrm{n}$ channel of the symmetric fusion reaction of ${ }^{89} \mathrm{Y}$ ions with ${ }^{92}$ Mo target nuclei. The beam, with an energy of $379 \mathrm{MeV}$ and an intensity of $\sim 15 \mathrm{pnA}$, was delivered by the Argonne Tandem Linac Accelerator System (ATLAS) at Argonne National Laboratory. The target was isotopically enriched 


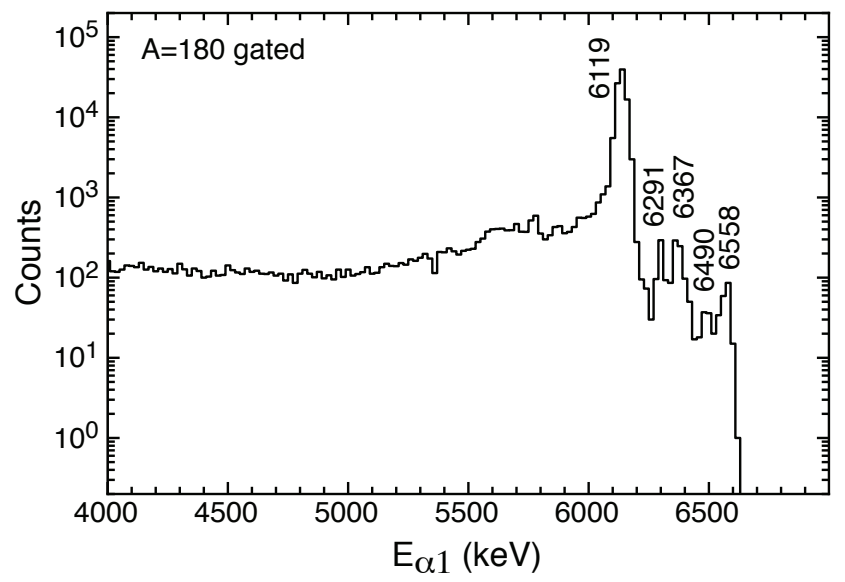

Figure 1. Energy spectrum of first-generation $\alpha$-decay events with a requirement that the decay occurred within $5 \mathrm{~s}$ of a mass $\mathrm{A}=180$ implant.

(>95\%) and self-supported with a thickness of approximately $510 \mu \mathrm{g} / \mathrm{cm}^{2}$.

Prompt $\gamma$ rays were detected with the Gammasphere array [12] consisting of 100 large volume escapesuppressed Ge detectors. The evaporation residues were transported through the Fragment Mass Analyzer (FMA) [13] and were dispersed according to their massto-charge $(\mathrm{m} / \mathrm{q})$ ratio. A position-sensitive parallel grid avalanche counter (PGAC), located at the FMA focal plane, provided the required $\mathrm{m} / \mathrm{q}$ information and the time of arrival of the recoils. The latter were subsequently implanted into a $160 \times 160$ strips $(\sim 142 \mu$ m thick $)$ doublesided silicon strip detector (DSSD). Each event in the DSSD was time stamped and identified as being either an implant or a charged-particle decay, depending on the coincidence or anti-coincidence with a signal from the PGAC. An array consisting of four CLOVER Ge detectors surrounded the DSSD. It was used to detect $\gamma$ rays in coincidence with $\alpha$-particle decays.

The ${ }^{180} \mathrm{Tl}$ residues and the corresponding prompt $\gamma$ rays were isolated from the dominant background originating from scattered beam, fission products, and deexcitations in neighboring isotopes produced in other reaction channels, by placing coincidence gates in the offline analysis on (i) the time of flight of the evaporation residues from the target to the focal plane, (ii) the PGAC positions corresponding to three charge states $(q=31,32$, and 33) of ions with the appropriate $A=180$ mass focus, and (iii) the two-dimensional histogram of the energy of recoils measured in the DSSD vs. the time of flight from the PGAC to the DSSD. The data were then sorted in coincidence histograms, gated in various ways on the energy and time information from the DSSD.

\section{Results and Discussion}

Figure 1 provides a first-generation $\alpha$-decay spectrum produced with the requirement that a $A=180$ recoil was im-

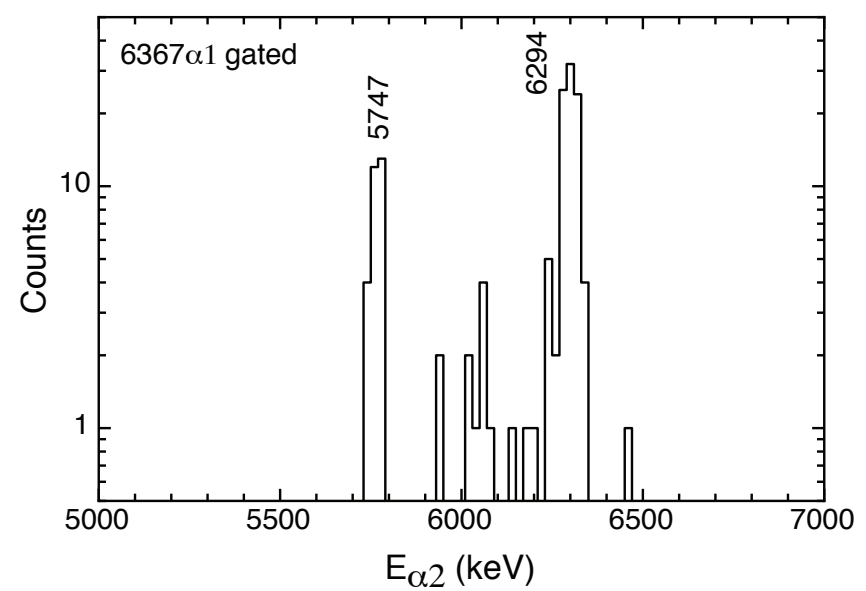

Figure 2. Energy spectrum of second-generation $\alpha$-decay events produced by gating on $\mathrm{E}_{\alpha 1}=6367 \mathrm{keV}$.

planted in the same pixel where a decay event was detected. The main line at $\mathrm{E}_{\alpha 1}=6119(5) \mathrm{keV}$ corresponds to the decay of ${ }^{180} \mathrm{Hg}$, which is produced in the $1 \mathrm{p}$ reaction channel. The higher-energy lines at $\mathrm{E}_{\alpha 1}=6291(10)$, 6367(10), 6490(10) and 6558(10) keV are associated with decays of ${ }^{180} \mathrm{Tl}$ (1n reaction channel). All of them were found to be correlated with $\mathrm{E}_{\alpha 2}=6294(10) \mathrm{keV}$ and $5747(10) \mathrm{keV} \alpha$ lines, as illustrated in Fig. 2. The former line is associated with the ground-state decay of the daughter nuclide, ${ }^{176} \mathrm{Au}$ [14], while the latter is identified as belonging to ${ }^{176} \mathrm{Pt}$ [15], produced in the EC/ $\beta^{+}$decay of ${ }^{176} \mathrm{Au}$. An $\alpha$-decay branching intensity of $\mathrm{b}_{\alpha}=75(8) \%$ was deduced for the ground-state decay of ${ }^{176} \mathrm{Au}$. It is worth noting that no correlations with the previously-known $\alpha$ lines associated with the decay of a high-spin isomer in ${ }^{176} \mathrm{Au}$ [14] were observed in the present work. This implies that only a single $\alpha$-decaying state exists in ${ }^{180} \mathrm{Tl}$. The half-life of ${ }^{180} \mathrm{Tl}$ was determined in the present work to be $T_{1 / 2}=1.1(2) \mathrm{s}$, in agreement with values reported in earlier studies [3, 9, 10]. A somewhat shorter value of $0.70(+12-9)$ s was reported by Lazarev et al. [2] from fission activity measurements. We have also measured a value of $\mathrm{T}_{1 / 2}=1.2(4) \mathrm{s}$ for the ground state of ${ }^{176} \mathrm{Au}$, in agreement with the earlier published results $[14,16]$. In addition, values of $\mathrm{T}_{1 / 2}=2.60(1) \mathrm{s}$ and 5.87(2) s were measured in the present work for ${ }^{180} \mathrm{Hg}$ and ${ }^{176} \mathrm{Pt}$, respectively.

Sample $\gamma$-ray spectra detected in the CLOVER array are presented in Fig. 3. The $\mathrm{E}_{\alpha 1}=6367 \mathrm{keV} \alpha$ decay line is observed to be in coincidence with only the 204.8(5)-keV $\gamma$ ray, while lines with energies of 69.9(5), $109.5(5), 204.8(5)$, and $210.7(5) \mathrm{keV}$ are in coincidence with $\mathrm{E}_{\alpha 1}=6291 \mathrm{keV}$. It is worth noting that 205- and 210$\mathrm{keV} \gamma$ rays were observed in the previous in-beam studies of ${ }^{176} \mathrm{Au}$ [14] and both were found to be correlated with the ground-state $\alpha$ decay. Based on the available $\alpha-\gamma$ coincidence information, a partial decay scheme of ${ }^{180} \mathrm{Tl}$ was constructed in the present work, as shown in Fig. 4. It should be noted, however, that we were not able to place 


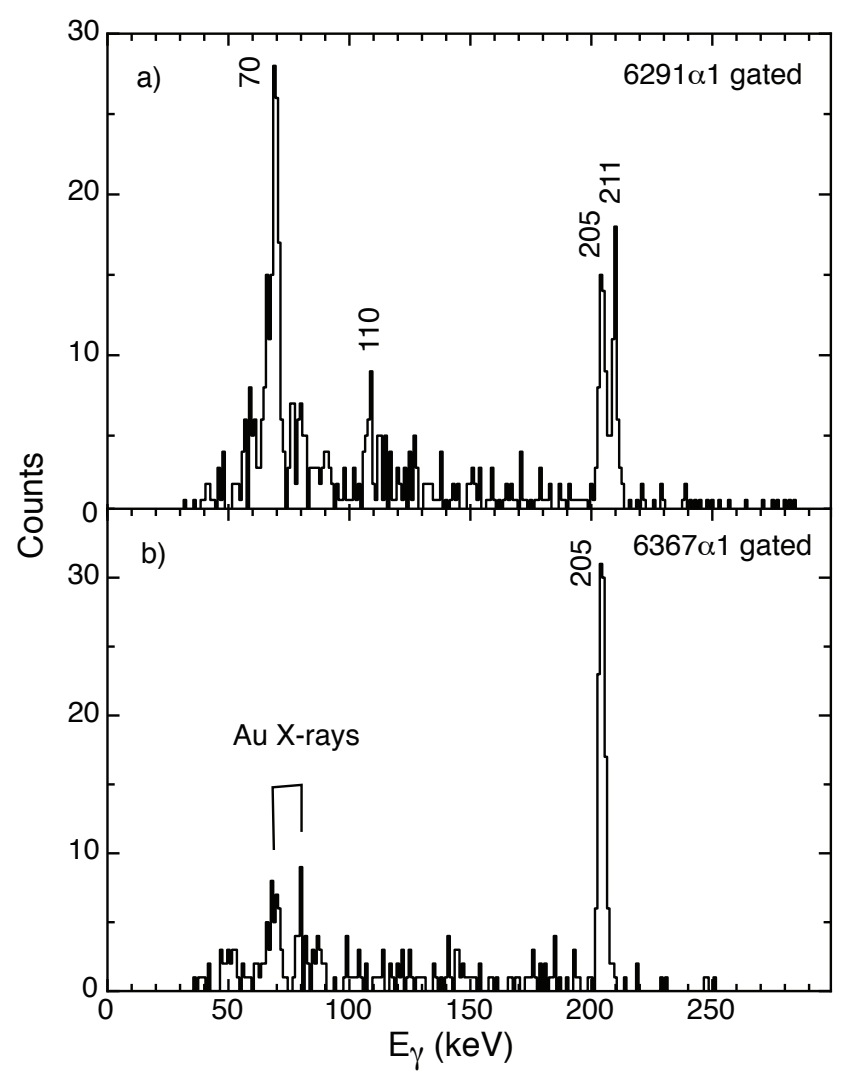

Figure 3. Gamma-ray spectra detected in the CLOVER array in coincidence with a) $\mathrm{E}_{\alpha 1}=6291 \mathrm{keV}$ and b) $\mathrm{E}_{\alpha 1}=6367 \mathrm{keV}$ decays.

the $109.5-\mathrm{keV} \gamma$ ray, as well as several other weak transitions, in the decay scheme.

Using the Recoil Decay Tagging method, we searched for in-beam $\gamma$ rays correlated with the ${ }^{180} \mathrm{Tl} \alpha$ decays. The statistics of resulting spectra were very low and we were able only to tentatively establish the 276-, 323-, 555-, and $1141-\mathrm{keV} \gamma$ rays as possible candidates. We were unable to confirm any of the three $\gamma$ rays that were reported in the $\alpha$-decay studies of ${ }^{184,184 m} \mathrm{Bi}$ [11]. This may be a consequence of the different level-population pattern in $\alpha$ decay, which is sensitive to the spin and configuration of the parent state that are presently unknown.

The neutron-deficient, odd - odd Tl nuclei with $\mathrm{N}$ $\geq 101$ are known to have a low-spin, $\mathrm{I}^{\pi}=2^{-}$, ground state and an $\mathrm{I}^{\pi}=7^{+}$spin-trap isomer $[17,18]$. Their structures can be interpreted as resulting from the coupling of the $\pi 1 / 2^{+}\left(\mathrm{s}_{1 / 2}\right)$ proton orbital, which is associated with the ground state of all even $-N \mathrm{Tl}$ isotopes, with the $v 3 / 2^{-}$ $\left(\mathrm{p}_{3 / 2}\right)$ and $v 13 / 2^{+}\left(\mathrm{i}_{13 / 2}\right)$ neutron orbitals, assigned to the ground and isomeric states in the odd $-N \mathrm{~Pb}$ nuclei, respectively $[17,18]$. It is worth noting that the systematic trend of the excitation energies of the $\mathrm{I}^{\pi}=7^{+}$isomers as a function of neutron number is very similar to that for the $v 13 / 2^{+}\left(\mathrm{i}_{13 / 2}\right)$ isomers in the odd $-N \mathrm{~Pb}$ isotopes. It has been shown for the first time by Carpenter et al. [4] that, at $\mathrm{N} \leq 99$, the structure of the $\mathrm{Pb}$ isotopes changes significantly and that the $v 9 / 2^{-}\left(\mathrm{h}_{9 / 2}\right)$ orbital becomes the ground

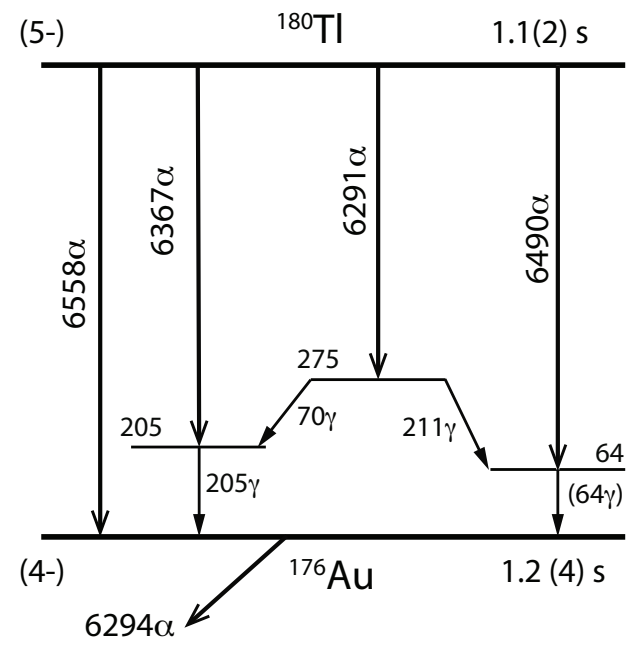

Figure 4. Partial $\alpha$-decay scheme of ${ }^{180} \mathrm{Tl}$ deduced in the present work.

state in ${ }^{181} \mathrm{~Pb}$. As a consequence, the ground state of ${ }^{180} \mathrm{Tl}$ $(\mathrm{N}=99)$ can be assigned $\mathrm{I}^{\pi}=5^{-}$with the $\pi 1 / 2^{+}\left(\mathrm{s}_{1 / 2}\right) \otimes$ $v 9 / 2^{-}\left(h_{9 / 2}\right)$ configuration. The spin assignment is supported by the observed direct populations of $\mathrm{I}=4,5$, and 6 levels in the ${ }^{180} \mathrm{Hg}$ daughter isotope, following $\mathrm{EC} / \beta^{+}$ decay of ${ }^{180} \mathrm{Tl}$ [9].

Changes in the neutron single-particle structure near $\mathrm{N}=99$ can also explain the absence of a long-lived isomeric state in ${ }^{180} \mathrm{Tl}$. The excitation energy of the $v 13 / 2^{+}\left(\mathrm{i}_{13 / 2}\right)$ level in ${ }^{181} \mathrm{~Pb}$ is unknown. However, if one extrapolates the known energies for this orbital from the heavier ${ }^{183} \mathrm{~Pb}$ $(\mathrm{N}=101),{ }^{185} \mathrm{~Pb}(\mathrm{~N}=103)$, and ${ }^{187} \mathrm{~Pb}(\mathrm{~N}=105)$ nuclei [18] towards $\mathrm{N}=99$, then the $\mathrm{I}^{\pi}=7^{+}, \pi 1 / 2^{+}\left(\mathrm{s}_{1 / 2}\right) \otimes v 13 / 2^{+}\left(\mathrm{i}_{13 / 2}\right)$ state in ${ }^{180} \mathrm{Tl}$ can be estimated to be located at $\sim 130 \mathrm{keV}$ and it can decay via an M2 transition to the $\mathrm{I}^{\pi}=5^{-}$ground state. A half-life of $\sim 5 \mu$ s can be expected for the $\mathrm{I}^{\pi}=7^{+}$ state using $\mathrm{B}(\mathrm{M} 2)=0.20$ (3) W.u., deduced from the decay of the $\mathrm{I}^{\pi}=13 / 2^{+}$isomer in ${ }^{179} \mathrm{Hg}(\mathrm{N}=99)$ [19], and a total electron conversion coefficient of $\alpha_{T} \sim 27.4$ [20] for a $130-\mathrm{keV}$, M2 transition in ${ }^{180} \mathrm{Tl}$. This value is much shorter than the expected partial $\alpha$ and $\mathrm{EC} / \beta^{+}$decays of $20 \mathrm{~s}$ and $1.3 \mathrm{~s}$, respectively, deduced from the known halflife and branching ratios for the ${ }^{180} \mathrm{Tl}$ ground state. Therefore, no $\alpha$ and/or $\mathrm{EC} / \beta^{+}$decays are expected from this $\mathrm{I}^{\pi}=7^{+}$state, which would rather de-excite via $\gamma$ rays and conversion electrons.

In contrast to ${ }^{180} \mathrm{Tl}$, both low- and high-spin $\alpha$ decaying states were observed in the daughter isotope ${ }^{176} \mathrm{Au}(\mathrm{N}=97)$ [14]. Since the $\pi 1 / 2^{+}\left(\mathrm{s}_{1 / 2}\right)$ proton orbital is assigned to the ground state of ${ }^{175} \mathrm{Au}[8,21]$ and the $v 7 / 2^{-}\left(\mathrm{h}_{9 / 2} / \mathrm{f}_{7 / 2}\right)$ neutron orbital to the ground state of ${ }^{177} \mathrm{Hg}(\mathrm{N}=97)$ [22], one may expect that the ground state of ${ }^{176} \mathrm{Au}$ has I${ }^{\pi}=4^{-}$with the $\pi 1 / 2^{+}\left(\mathrm{s}_{1 / 2}\right) \otimes v 7 / 2^{-}\left(\mathrm{h}_{9 / 2} / \mathrm{f}_{7 / 2}\right)$ configuration. A coupling between the $v 13 / 2^{+}\left(\mathrm{i}_{13 / 2}\right)$ and $\pi 1 / 2^{+}\left(\mathrm{s}_{1 / 2}\right)$ orbitals can result in a higher-spin $\mathrm{I}^{\pi}=7^{+}$state in ${ }^{176} \mathrm{Au}$. However, it cannot account for the long-lived nature of the isomer. For example, based on the known energy of the $v 13 / 2^{+}\left(\mathrm{i}_{13 / 2}\right)$ orbital in ${ }^{177} \mathrm{Hg}(\mathrm{N}=97)$ [22], this 
$\mathrm{I}^{\pi}=7^{+}$state would be expected at $\sim 320 \mathrm{keV}$ in ${ }^{176} \mathrm{Au}$ and it could decay via an E3 $\gamma$-ray transition to the $\mathrm{I}^{\pi}=4^{-}$ground state. Using $\mathrm{B}(\mathrm{E} 3) \approx 22$ W.u. [23] and $\alpha_{T}(\mathrm{E} 3)=0.455$ [20] for a 320-keV, E3 transition, a value of $\mathrm{T}_{1 / 2} \sim 60 \mu \mathrm{s}$ can be estimated for the $\mathrm{I}^{\pi}=7^{+}$state. This is much shorter than the measured $\mathrm{T}_{1 / 2}=1.36(2) \mathrm{s}$ [14] for the isomer.

A high-spin, $\mathrm{I}^{\pi}=11 / 2^{-}$isomer associated with the proton $\left(\mathrm{h}_{11 / 2}\right)$ orbital is observed in the neighboring ${ }^{175} \mathrm{Au}$ $(\mathrm{N}=96)$ and ${ }^{177} \mathrm{Au}(\mathrm{N}=98)$ isotopes at 207(14) and 189(8) $\mathrm{keV}$, respectively $[8,18]$. Coupling of this orbital with the $v 7 / 2^{-}\left(\mathrm{h}_{9 / 2} / \mathrm{f}_{7 / 2}\right)$ state would result in a high-spin $\mathrm{I}^{\pi}=9^{+}$ level, located at relatively low energy, $200 \mathrm{keV}$. A $\gamma$-ray decay via an M5 transition to the $\mathrm{I}^{\pi}=4^{-}$ground state and the competing emission of conversion electrons would be severely retarded $\left(\mathrm{T}_{1 / 2} \sim 7 \times 10^{5} \mathrm{~d}\right)$ and, hence, the decay can only proceed via $\alpha$ and/or EC/ $\beta^{+}$decays. Therefore, the $\alpha$-decaying isomer in ${ }^{176} \mathrm{Au}$ can be interpreted as a spin trap and assigned $\mathrm{I}^{\pi}=9^{+}$with the $\pi 11 / 2^{-}\left(\mathrm{h}_{11 / 2}\right) \otimes v 7 / 2^{-}$ $\left(\mathrm{h}_{9 / 2} / \mathrm{f}_{7 / 2}\right)$ configuration. It should be noted that the $\pi 11 / 2^{-}$ $\left(\mathrm{h}_{11 / 2}\right)$ state is at much higher excitation energy (above 800 $\mathrm{keV}$ ) in the even $-N \mathrm{Tl}$ isotopes [4, 8, 21, 24], and, hence, one may expect that it would not result in a long-lived isomer in the case of ${ }^{180} \mathrm{Tl}$.

\section{Acknowledgements}

This work was supported by the U.S. Department of Energy, Office of Nuclear Physics, under Contract No. DE-AC0206CH11357 and Grant No. DE-FG02-94-ER40834, and the U.S. National Science Foundation, under Grant No. PHY-1203100.

\section{References}

[1] K. Heyde and J.L. Wood, Rev. Mod. Phys. 83, 1467 (2011).

[2] Yu. A. Lazarev, Yu. Ts. Oganessian, I. V. Shirokovsky, S. P. Tretyakova, V. K. Utyonkov, and G. V. Buklanov, Europhys. Lett. 4, 893 (1987).

[3] A. N. Andreyev et al., Phys. Rev. Lett. 105, 252502 (2010).

[4] M.P. Carpenter, F.G. Kondev, and R.V.F. Janssens, J. Phys. G31, S1599 (2005).

[5] M.P. Carpenter et al., AIP Conf. Proc. 1098, 58 (2009).
[6] W. Mang, G. Audi, A.H. Wapstra, F.G. Kondev, M. MacCormick, X. Xu, and B. Pfeiffer, Chin. Phys. C 36, 1603 (2012).

[7] M. Dasgupta, D.J. Hinde, N. Rowley, and A.M. Stefanini, Annu. Rev. Nucl. Part. Sci. 48, 401 (1998).

[8] C. Nair et al., Phys. Rev. C (submitted for publication).

[9] J. Elseviers et al., Phys. Rev. C84, 034307 (2011).

[10] K. Toth et al., Phys. Rev. C58, 1310 (1988).

[11] A. N. Andreyev et al., Eur. Phys. J. A18, 55 (2003).

[12] R. V. F. Janssens and F. S. Stephens, Nuclear Physics News 6, 9 (1996).

[13] C. N. Davids et al., Nucl. Instrum. Methods B70, 358 (1992).

[14] J. TM. Goon, PhD Thesis, University of Tennessee, 2004 (unpublished).

[15] G. Audi, W. Mang, A.H. Wapstra, F.G. Kondev, M. MacCormick, X. Xu, and B. Pfeiffer, Chin. Phys. C 36, 1287 (2012).

[16] P.M. Davidson, G.D. Dracoulis, T.Kibédi, A.P. Byrne, S.S. Anderssen, A.M. Baxter, B. Fabricius, G.J. Lane, and A.E. Stuchbery, Nucl. Phys. A657, 219 (1999).

[17] Evaluated Nuclear Structure Data File (www.nndc.bnl.gov/ensdf).

[18] G. Audi, F.G. Kondev, W. Mang, B. Pfeiffer, X. Sun, J. Blachot, and M. MacCormick, Chin. Phys. C 36, 1157 (2012).

[19] D.G. Jenkins et al., Phys. Rev. C66, 011301(R) (2003).

[20] T. Kibédi, T.W. Burrows, M.B. Trzhaskovskaya, P.M. Davidson, and C.W. Nestor, Jr., Nucl. Instr. and Meth. A 589, 202 (2008).

[21] G.L. Poli et al., Phys. Rev. C59, R2979 (1999).

[22] A. Melerangi et al., Phys. Rev. C68, 041301(R) (2003).

[23] G. D. Dracoulis, T. Kibédi, A. P. Byrne, A. M. Baxter, S. M. Mullins, and R. A. Bark, Phys. Rev. C63, 061302(R) (2001).

[24] M. Muikku et al., Phys. Rev. C64, 044308 (2001). 